\title{
The Mean-CVaR Model for Portfolio Optimization Using a Multi-Objective Approach and the Kalai-Smorodinsky Solution
}

\author{
R. Aboulaich ${ }^{1}, R$. Ellaia ${ }^{1}, S$. Elmoumen ${ }^{2}, A$. Habbal $^{3}$, and N. Moussaid ${ }^{4, \star}$ \\ ${ }^{1}$ LERMA, Mohammadia School of Engineering, Mohammed V University Rabat, Morocco \\ ${ }^{2}$ LIMSAD, Mathematics and computing Department, Ain Chock Sciences Faculty, Casablanca, Morocco \\ ${ }^{3}$ INRIA, University of Nice Sophia-Antipolis, France \\ ${ }^{4}$ LMA, Hassan II University Casablanca, FST, Mohammedia, Morocco
}

\begin{abstract}
The purpose of this work is to present a model for portfolio multi-optimization, in which distributions are compared on the basis of tow statistics: the expected value and the Conditional Value-at-Risk (CVaR), to solve such a problem many authors have developed several algorithms, in this work we propose to find the efficient boundary by using the Normal Boundary Intersection approach (NBI) based on our proposed hybrid method SASP, since the considered problem is multi-objective, then we find the Kalai-smorodinsky solution.
\end{abstract}

\section{Introduction}

Mean-risk models are still the most widely used approach in the practice of portfolio selection. With mean-risk models, return distributions are characterized and compared using two statistics: the expected value and the value of a risk measure. Thus, mean-risk models have a ready interpretation of results and in most cases are convenient from a computational point of view. On the other hand in the practice of describing a distribution by just two parameters involves great loss of information.

It is well known that the risk measure plays an important role in making the decisions. Variance was the first risk measure used in mean-risk models (Markowitz 1952) and, in many proposals of new risk measures (see for example Fishburn (1977), Yitzhaki (1982), Konno and Yamazaki (1991), Ogryczak and Ruszczynski (1999, 2001), Rockafellar and Uryasev (2000, 2002)). Another risk measure, the Conditional Value-at-Risk (CVaR))[16],[20], is growing in popularity. CVaR has attractive theoretical properties: it controls the magnitude of losses beyond Valueat-Risk (VaR) and it is coherent (see for example Artzner 1999, Acerbi and Tasche 2002, Tasche 2002, Pflug 2000, Rockafellar and Uryasev 2002). In this paper we propose to solve the problem of portfolio selection, which is a multi-objective problem, first by using the NBI approach [8] based on SASP method [7], implemented in Matlab to find the efficient boundary, after we use the game theory $[3,4][9][2][1]$ to determine the Kalai-smorodinsky solution.

\section{The portfolio selection problem}

The problem of portfolio selection with one investment period is an example of the general problem of deciding between random variables when larger outcomes are preferred. Decisions are required on the amount (proportion) of capital to be invested in each of a number of available assets such that at the end of the investment period the return is as high as possible. Consider a set of $n$ assets, with asset $j$ in $\{1, \ldots, n\}$ giving a return $R_{j}$ at the end of the investment period. $R_{j}$ is a random variable, since the future price of the asset is not known. Let $x_{j}$ be the proportion of capital invested in asset $j\left(x_{j}=w_{j} / w\right.$ where $w_{j}$ is the capital invested in asset $j$ and $w$ is the total amount of capital to be invested), and let $x=\left(x_{1}, \ldots, x_{n}\right)$ represent the portfolio resulting from this choice. This portfolio's return is the random variable: $R_{x}=x_{1} R_{1}+\ldots+x_{n} R_{n}$, with distribution function $F(r)=P\left(R_{x} \leq r\right)$ that depends on the choice $x=\left(x_{1}, \ldots, x_{n}\right)$.

To represent a portfolio, the weights $\left(x_{1}, \ldots, x_{n}\right)$ must satisfy a set of constraints that forms a feasible set $\mathbf{A}$ of decision vectors. The simplest way to define a feasible set is by the requirement that the weights must sum to 1 and short selling is not allowed. For this basic version of the problem, the set of feasible decision vectors is:

$$
\mathbf{A}=\left\{\left(x_{1}, \ldots, x_{n}\right) / \sum_{i=1}^{n} x_{j}=1, x_{j} \geq 0, \forall j \in\{1, \ldots, n\}\right\}
$$

If we consider a different portfolio defined by the decision vector $y=\left(y_{1}, \ldots, y_{n}\right) \in \mathbf{A}$, where $y_{j}$ is the proportion of capital invested in asset $j$. The return of this portfolio is given by the random variable $R_{y}=y_{1} R_{1}+\ldots+y_{n} R_{n}$.

The problem of choosing between portfolio $x=\left(x_{1}, \ldots, x_{n}\right)$ and portfolio $y=\left(y_{1}, \ldots, y_{n}\right)$ becomes the problem of

\footnotetext{
^e-mail: moussaid_noureddine@yahoo.fr
} 
choosing between random variables $R_{x}$ and $R_{y}$. The criteria by which one random variable is considered "better" than another random variable need to be specified and models for choosing between random variables (models for preference) are required. a lot of models are proposed, the markowitz model, the mean model and the mean variance model.

\section{Conditional Value-at-Risk}

Let $R_{x}$ be a random variable representing the return of a portfolio $x$ over a given holding period and $\alpha \in(0,1)$ a percentage which represents a sample of "worst cases" for the outcomes of $R_{x}$.

The definition of CVaR at the specified level $\alpha$ is the mathematical transcription of the concept "average of losses in the worst $\alpha$ of cases" (Acerbi and Tasche 2002), where a "loss" is a negative outcome of $R_{x}$ (thus the loss associated with $R_{x}$ is described by the random variable $-R_{x}$ ). An important result, proved by Rockafellar and Uryasev (2000, 2002), and independently by Ogryczak and Ruszczynski (2002), is that the CVaR of a random variable $R_{x}$ can be calculated by solving an optimization problem. Moreover, CVaR can be minimized over the set of feasible decision vectors. These results are summarized below:

Let $R_{x}$ be a random variable depending on a decision vector $x$ that belongs to a feasible set $\mathbf{A}$, and $\alpha \in(0,1)$. Consider the function:

$$
F_{\alpha}(x, v)=\frac{1}{\alpha} E\left\{\left[-R_{x}+v\right]^{+}\right\}-v
$$

Then:

(a) As a function of $v, F_{\alpha}$ is finite and continuous (hence convex) and

$$
C V a R_{\alpha}\left(R_{x}\right)=\min _{v \in R} F_{\alpha}(x, v)
$$

(b) Minimizing $C V a R_{\alpha}$ with respect to $x \in A$ is equivalent to minimizing $F_{\alpha}$ with respect to $(x, v) \in A \times R$ :

$$
\min _{x \in A} C \operatorname{VaR} R_{\alpha}\left(R_{x}\right)=\min _{(x, v) \in A \times R} F_{\alpha}(x, v) .
$$

(c) $C \operatorname{VaR} R_{\alpha}\left(R_{x}\right)$ is convex with respect to $x$ and $F_{\alpha}(x, v)$ is convex with respect to $(x, v)$.

\section{The Mean-CVaR model}

Let $E$ be the expected value of $R_{x}$, the Mean-CVaR model can be formulated for the portfolio selection problem as follows:

$$
\min _{x \in A} F=\left(f_{1}, f_{2}\right)
$$

Where $f_{1}=C V a R$ and $f_{2}=-E$

Since 1, the problem 2 becomes:

$$
\min _{x \in A}\left(F_{\alpha}(x, v),-E\right)
$$

\section{Normal Boundary Intersection}

Let $x_{i}^{*}$ and $f_{i}^{*}$ denote respectively the minimizer and minimum value of the $f_{i}$ and let $F^{*}$ denote the shadow minimum, i.e., the vector whose components are $f_{i}^{*}$. Consider the shifted pay-off matrix $\Phi$ whose $i^{t} h$ column is $F\left(x_{i}^{*}\right)-F^{*}$. The Convex Hull of Individual Minima or CHIM is defined as the set of points that are convex combinations of the columns of $\Phi$, i.e., $\left\{\Phi \beta: \beta_{i} \geq 0, \sum_{i} \beta_{i}=1\right\}$.

For a two dimensional problem illustrated in Figure 1, $\mathrm{CHIM}$ is represented by segment $\mathrm{AB}$.

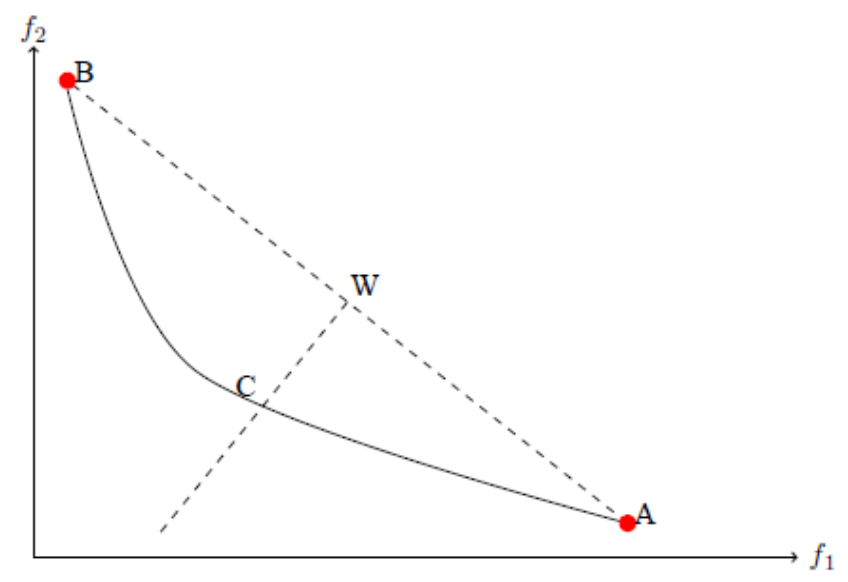

Figure 1. An illustrative integrated design

The idea behind NBI is to pick an even spread of points on the CHIM (for example $\mathrm{W}$ in Fig.1), and find the intersection point between the efficient frontier and a set of parallel normals emanating from the chosen set of points on the CHIM (C in Fig.1). Given a convex combination parameter vector $\beta$, and a normal direction $\mathrm{n}$ pointing towards the origin, the point of intersection between the normal emanating from $\Phi \beta$ and the efficient frontier can be found by solving the following $N B I_{\beta}$ subproblem :

$$
\left\{\begin{array}{l}
\min _{x \in A} t \\
\Phi \beta+t n=F(x)-F^{*}
\end{array}\right.
$$

By solving subproblem $N B I_{\beta} 4$ for different settings of $\beta$, various points on the efficient frontier can be generated. The advantage of the $\beta$ parameter is that an even spread of $\beta$ parameters corresponds to an even sped of points on the CHIM.

\section{Kalai Smorodinsky solution}

In this paper, we research the Kalai Smorodinsky solution [18], witch is the intersection between the Pareto front and the line passing through the utopian point $U t$ and the disagreement point $D$. Note,

$$
U t=\left(\begin{array}{c}
f_{1}\left(x^{*}\right) \\
f_{2}\left(y^{*}\right)
\end{array}\right), D=\left(\begin{array}{c}
f_{1}\left(y^{*}\right) \\
f_{2}\left(x^{*}\right)
\end{array}\right) \text { and } \tau=\frac{U t-D}{\|U t-D\|}
$$


where,

$$
\left\{\begin{array}{c}
x^{*}=\operatorname{Arg} \min _{x} f_{1}(x) \\
y^{*}=\operatorname{Arg} \min _{y} f_{2}(y) .
\end{array}\right.
$$

Theorem 1 A solution is symmetric, Pareto optimal[9][2][1], invariant under affine transformations of utility scale, and individually monotonic if and only if it is the Kalai-Smorodinsky solution [18].

To search the Kalai Smorodinsky solution [5], we proposed to solve the following problem:

$$
(K S)\left\{\begin{array}{l}
\max _{y, t} t, \\
\text { s.c } F(x)=D+t \tau,
\end{array}\right.
$$

To solve such problem we propose to use the SASP method [7].

\section{Numerical Example}

\subsection{An application to portfolio optimization}

Let (SAMIR, ATWB, AGM, LAM, GAZ, IAM, ONA) be the assets, quoted in the Casablanca Stock Exchange, with history of 5 years (see the following Figure).

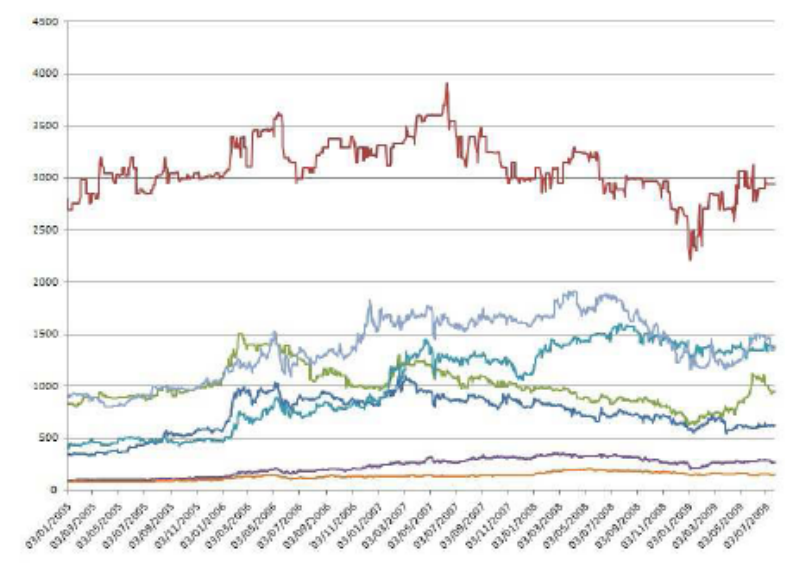

Figure 2. An illustrative integrated design

First we are going to solve the problems (8) and (9):

$$
\min _{x \in A} C V a R
$$

and

$$
\min x \in A-E
$$

Then we have to find the efficient frontier with the NBI method based on the SASP method, for this, we have to solve the problems below for deferent $\beta$ with $\beta \in\{\beta \in$ $\left.I R^{n}, \sum_{i=1}^{n} \beta_{i}=1, \beta_{i} \geq 0\right\}$ :

$$
\begin{gathered}
\max \quad t \\
\text { s.t. } \Phi \beta+t n=F(x)-F^{*} \\
x \in A
\end{gathered}
$$

The Kalai Smorodinsky solution can be find by solving the following problem:

$$
(K S)\left\{\begin{array}{l}
\max _{x, t} t, \\
\operatorname{s.c} F(x)=D+t \tau,
\end{array}\right.
$$

\subsection{Results}

The results are provided by the following figure:

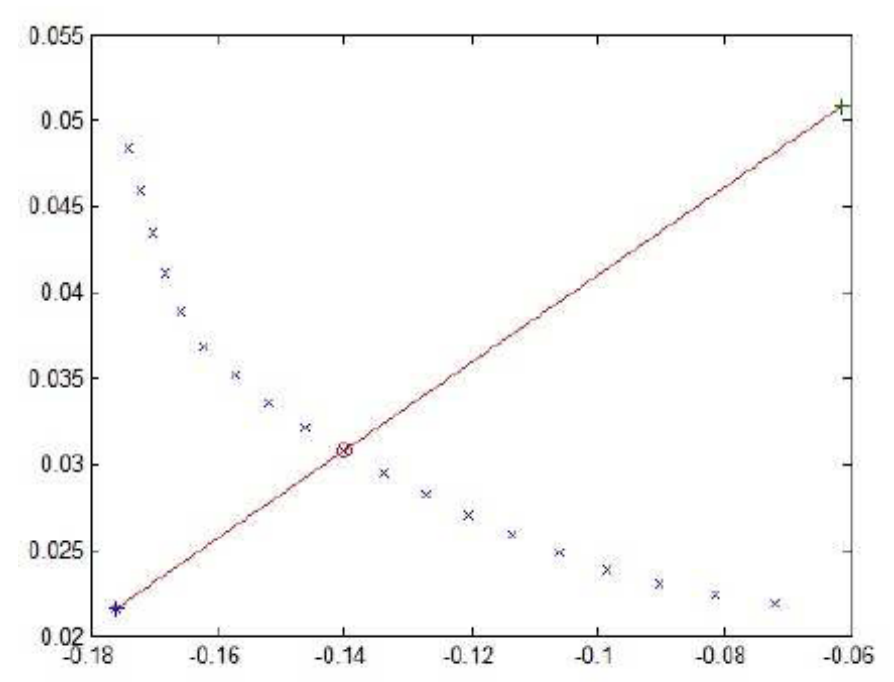

Figure 3. An illustrative integrated design

As we can see clearly the NBI parametrization of the efficient front naturally bears the property of even spread, and the Kalai Smorodinsky solution is closest to the ideal solution (maximum E, minimum CVaR), the Kalai Smorodinsky portfolio "PF" (36\% in ATW, 37\% in GAZ and $27 \%$ in IAM) history is given with the other histories in the following figure:

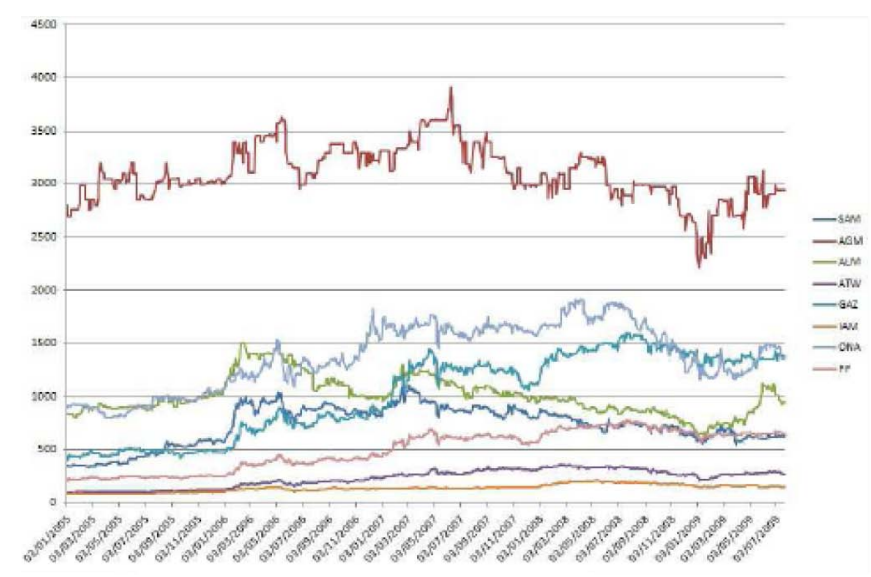

Figure 4. An illustrative integrated design

If we choose another optimal portfolio, for example the portfolio corresponding to $(0,1061,0,0248)$ we ob- 
tain $(13,81 \%$ in AGM,5,02\% ALM, 22,93\% ATW, 25,5\% GAZ and 32,75 IAM) and for $(0,1722,0,0459)$ we obtain (14,21\% ATW, 85,79\% GAZ), we can't have an equilibrium between maximum $\mathrm{E}$ and minimum $\mathrm{CVaR}$.

\section{Conclusion}

In this work, we proposed a solution of bi-objective model in order to obtain the optimal portfolio. First we used the Normal Boundary Intersection approach (NBI) to compute the efficient front, and then the optimal solution is given by Kalai Smorodinsky solution. The obtained results show the efficiency of the proposed approach.

\section{References}

[1] B. Abou El Majd, J.A. Désidéri, and A. Habbal. Aerodynamic and structural optimization of a business-jet wingshape by a Nash game and an adapted split of variables. Mécanique \& Industries 11.3-4 209-214, (2010).

[2] B. Abou El Majd. Optimisation de forme paramétrique: Stratégies hiérarchiques, adaptatives, et concourantes. Éditions universitaires européennes, (2015).

[3] R. Aboulaich, A. Habbal and N. Moussaid, Split of an optimization variable in game theory, Math. Model. Nat. Phenom(MMNP) 5, No. 7, 106-111,(2010).

[4] R. Aboulaich, A. Habbal and N. Moussaid, Optimisation multicritère : une approche par partage des variables, 13, 80-87,(2010).

[5] R. Aboulaich, R. Ellaia, S. El moumen, A. Habbal, N. Moussaid. A new algorithm for approching Nash equilibrium and Kalai Smoridinsky solution, submitted to "Applied and Computational Mathematics an International Journal (ACMIJ)".

[6] R. Aboulaich, R. Ellaia, S. El moumen. The MeanVariance-CVaR model for portfolio optimization Modeling using a Multi-Objective Approach based on a hybrid method, Math. Model. Nat. Phenom, 7, 93-98, (2010).

[7] R. Aboulaich, R. Ellaia, S. El Moumen. A new hybrid method for solving global optimization problem, Applied Mathematics and Computation Volume 218, Issue 7, 3265-3276, (2011).

[8] I. Das, J.E. Dennis. Normal Boundary Intersection, A New methode for Generating the Pareto Surface in
Nonlinear Multicreteria Optimization problems, SIAM J. Optimization, 3, 631-657, (1998).

[9] J. A. Désidéri, R. Duvigneau, B. Abou El Majd, and Z. Tang. Algorithms for efficient shape optimization in aerodynamics and coupled disciplines. In 42nd AAAF Congress on Applied Aerodynamics, March 2007, Sophia-Antipolis, France.

[10] M. Duran Toksarý. A heuristic approach to find the global optimum of function, Journal of Computational and Applied Mathematics 209 160-166, (2007).

[11] S. Kirpatrick, C.D. Gelatt, and M.P. Vecchi. Optimization by simulated annealing, Science,New Series 220), 671-680, (1983).

[12] H.M. Markowitz. Multivariate Stochastic Approximation using a Simultaneous Perturbation Gradient Approximation, 7, 77-91, (1952).

[13] N. Metropolis, A.W. Rosenbluth, M.N. Rosenbluth, A.H. Teller, and E. Teller. Equations of state calculations by fast computing machines, J. Chem. Phys., 21(6), 1087-1091,(1953).

[14] B. Raphael , I.F.C. Smith. A direct stochastic algorithm for global search, Applied Mathematics and Computation 146, 729-758,(2003).

[15] C.R. Reeves. Modern Heuristic Techniques for Combinatorial Problems, John Wiley and Sons, New York, NY, 1993.

[16] R.T. Rockafeller, S. Uryasev. Optimization of Conditional Value-at-Risk, Journal of Risk,3, 21-42, (2000).

[17] R.T. Rockafeller, S. Uryasev. Conditional Value-atRisk for general loss distributions, Journal of Banking and Finance, 7, 1443-1471, (2002).

[18] M. Smorodinsky, E. Kalai. Other Solution to Nash's Bargaining Problem, Econometrica, Applied Mathematics and Computation, 3, 513-518, (1975).

[19] J.C. Spall. Multivariate Stochastic Approximation using a Simultaneous Perturbation Gradient Approximation, IEEE transactions on automatic control, 37 332-341, (1992).

[20] S. Uryasev. Conditional Value-at-Risk: Optimization Algorithms and Applications, Financial Engineering News 14, 1-5, (2000).

[21] Q. Yuan, Z. He, H. Leng. A hybrid genetic algorithm for a class of global optimization problems with box constraints, Applied Mathematics and Computation 197 (2008) 924-929. 